\title{
Effectiveness and safety of nivolumab in patients with head and neck cancer in Japanese real-world clinical practice: a multicenter retrospective clinical study
}

\author{
Nobuhiro Hanai ${ }^{1} \cdot$ Yasushi Shimizu $^{2} \cdot$ Shin Kariya ${ }^{3} \cdot$ Ryuji Yasumatsu $^{4} \cdot$ Tomoya Yokota $^{5} \cdot$ Takashi Fujii $^{6}$. \\ Kiyoaki Tsukahara ${ }^{7}$ Masafumi Yoshida ${ }^{8} \cdot$ Kenji Hanyu $^{9} \cdot$ Tsutomu Ueda $^{10} \cdot$ Hitoshi Hirakawa $^{11}$. Shunji Takahashi ${ }^{12}$. \\ Takeharu Ono ${ }^{13}$. Daisuke Sano ${ }^{14} \cdot$ Moriyasu Yamauchi ${ }^{15}$. Akihito Watanabe ${ }^{16} \cdot$ Koichi Omori $^{17}$. \\ Tomoko Yamazaki ${ }^{18} \cdot$ Nobuya Monden $^{19} \cdot$ Naomi Kudo $^{20} \cdot$ Makoto Arai $^{21} \cdot$ Daiju Sakurai $^{22} \cdot$ Takahiro Asakage $^{23}$. \\ Issei Doi ${ }^{24} \cdot$ Takayuki Yamada $^{25} \cdot$ Akihiro Homma ${ }^{26}$
}

Received: 25 June 2020 / Accepted: 29 October 2020

(c) The Author(s) 2020, corrected publication 2021

\begin{abstract}
Background To fill the data gap between clinical trials and real-world settings, this study assessed the overall effectiveness and safety of nivolumab in patients with head and neck cancer (HNC) during Japanese real-world clinical practice.

Methods This was a multicenter, retrospective study in Japanese patients with recurrent or metastatic HNC who received nivolumab for the first time between July and December 2017. Data on the clinical use, effectiveness, and safety of nivolumab were extracted from patient medical records.

Results Overall, 256 patients were enrolled in this study. The median duration of nivolumab treatment was 72.5 days, with patients receiving a median of 6.0 (range 1-27) doses. Median overall survival (OS) was 9.5 (95\% confidence interval [CI] 8.2-12.0) months and the estimated 12 -month OS rate was $43.2 \%$. The objective response rate (ORR) was $15.7 \%$ overall and $21.1 \%, 7.1 \%$, and $13.6 \%$ in patients with primary nasopharynx, maxillary sinus, and salivary gland tumors, respectively, who had been excluded from CheckMate 141. Grade $\geq 3$ immune-related adverse events occurred in $5.9 \%$ of patients. No new safety signals were identified compared with adverse events noted in CheckMate 141.

Conclusions The effectiveness and safety of nivolumab in real-world clinical practice are consistent with data from the CheckMate 141 clinical trial. Therapeutic response was also observed in the groups of patients excluded from CheckMate 141.

Trial registration number UMIN-CTR (UMIN000032600), Clinicaltrials.gov (NCT03569436)
\end{abstract}

Keywords Nivolumab $\cdot$ Real-world clinical practice $\cdot$ Recurrent or metastatic head and neck cancer $\cdot$ Multicenter retrospective study

\section{Introduction}

Nivolumab is a fully human immunoglobulin G4 monoclonal antibody targeted against programmed cell death protein-1 (PD-1). Nivolumab was approved in March 2017 for the treatment of recurrent or distant metastatic head and

Electronic supplementary material The online version of this article (https://doi.org/10.1007/s10147-020-01829-0) contains supplementary material, which is available to authorized users.

Akihiro Homma

ak-homma@med.hokudai.ac.jp

Extended author information available on the last page of the article neck cancer (HNC) in Japan. This approval was based on the survival benefits and the manageable safety profile demonstrated by nivolumab in the global phase III CheckMate 141 study, also known as ONO-4538-11 [1]. CheckMate 141 showed that nivolumab significantly prolonged overall survival (OS) compared with standard therapy alone in patients with recurrent squamous cell HNC (median OS of 7.5 vs 5.1 months; $p=0.01$ ) [1]. In addition, OS benefit was maintained with 2-year follow-up [2].

In general, patients eligible for clinical trials are highly selected [3]. To be eligible for CheckMate 141, patients were required to have platinum-refractory squamous cell HNC. CheckMate 141 also excluded patients with Eastern Cooperative Oncology Group performance status (ECOG PS) $\geq 2$. 
Table 1 Baseline characteristics of patients

\begin{tabular}{|c|c|}
\hline Characteristics & Nivolumab $(N=256)$ \\
\hline \multicolumn{2}{|l|}{ Sex, $n(\%)$} \\
\hline Male & $202(78.9)$ \\
\hline Female & $54(21.1)$ \\
\hline \multicolumn{2}{|l|}{ Age, years } \\
\hline Median (range) & $66(20-84)$ \\
\hline Age $\geq 75$ years, $n(\%)$ & $24(9.4)$ \\
\hline \multicolumn{2}{|l|}{ Primary cancer site, $n(\%)$} \\
\hline Hypopharynx & $64(25.0)$ \\
\hline Oral cavity & $56(21.9)$ \\
\hline Oropharynx & $40(15.6)$ \\
\hline Salivary glands* & $23(9.0)$ \\
\hline Larynx & $21(8.2)$ \\
\hline Nasopharynx* & $19(7.4)$ \\
\hline Maxillary sinus* & $14(5.5)$ \\
\hline Others* & $19(7.4)$ \\
\hline \multicolumn{2}{|c|}{ Presence of another primary malignancy, $n(\%)$} \\
\hline Yes* & $49(19.1)$ \\
\hline No & $199(77.7)$ \\
\hline Unknown & $8(3.1)$ \\
\hline \multicolumn{2}{|c|}{ Head and neck cancer histology, $n(\%)$} \\
\hline Squamous cell carcinoma & $217(84.8)$ \\
\hline Non-Squamous cell carcinoma* & $29(11.3)$ \\
\hline Not Evaluated* & $9(3.5)$ \\
\hline \multicolumn{2}{|l|}{ ECOG PS, $n(\%)$} \\
\hline 0 & $118(46.1)$ \\
\hline 1 & $97(37.9)$ \\
\hline $2 *$ & $23(9.0)$ \\
\hline $3^{*}$ & $6(2.3)$ \\
\hline $4 *$ & $2(0.8)$ \\
\hline Unknown* & $10(3.9)$ \\
\hline \multicolumn{2}{|c|}{ Cancer stage at nivolumab initiation, $n(\%)$} \\
\hline $\mathrm{I}^{*}$ & $11(4.3)$ \\
\hline $\mathrm{II}^{*}$ & $30(11.7)$ \\
\hline III & $22(8.6)$ \\
\hline IVA & $114(44.5)$ \\
\hline IVB & $29(11.3)$ \\
\hline IVC & $33(12.9)$ \\
\hline Unknown* & $17(6.6)$ \\
\hline \multicolumn{2}{|c|}{$\begin{array}{l}\text { Nivolumab treatment line for recurrent or metastatic head and neck } \\
\text { cancer, } n(\%)^{* *}\end{array}$} \\
\hline $1 \mathrm{st}$ & $70(27.3)$ \\
\hline 2nd & $110(43.0)$ \\
\hline 3 rd & $45(17.6)$ \\
\hline 4th or later & $31(12.1)$ \\
\hline
\end{tabular}

ECOG Eastern Cooperative Oncology Group

*Patients with these characteristics were excluded from the CheckMate 141 study [1]

** Nivolumab treatment line was counted as the number of systemic chemotherapy for recurrent or metastatic HNC
Fig. 1 a Overall response rate b progression-free survival and $\mathbf{c}$ overall survival among all patients. $C I$ confidence intervals, $B O R$ best overall response, $C R$ complete response, $O R R$ objective response rate, $O S$ overall survival, $P D$ progressive disease, $P F S$ progression-free survival, $P R$ partial response, $S D$ stable disease

In addition, the CheckMate 141 study specifically excluded patients with primary tumor sites such as the nasopharynx, nasal cavity, paranasal sinuses, salivary glands, or lip [1].

In melanoma and non-small cell lung cancer, nivolumab has shown long-term survival in patients with stable disease (SD) as well as complete response (CR) or partial response (PR) $[4,5]$. In HNC patients, however, the effectiveness by the best overall response (BOR) has not been reported in real-world practice.

In Japan, nivolumab is indicated for any type of HNC, and is not restricted to only those subtypes included in the CheckMate 141 study. In addition, only 18 patients from Japan received nivolumab in CheckMate 141. This means that there is a data gap between the results of the clinical trial and how nivolumab performs in real-world clinical practice in Japan.

The aim of this observational study was to evaluate the effectiveness and safety of nivolumab in a large real-world setting in Japan, and to address the data gap between clinical trial and real-world settings. Here, we report the primary results of a retrospective chart review of patients with HNC who were treated with nivolumab in Japan.

\section{Patients and methods}

\section{Study design and patients}

This was a multicenter, non-interventional, retrospective study conducted at 23 centers in Japan in accordance with relevant regulations in Japan (Ministerial Ordinance on Good Post-Marketing Study Practice, Ministry of Health, Labour and Welfare Ordinance Number 171, December 20, 2004). The study protocol was reviewed and approved by the Institutional Review Board/Independent Ethics Committee at each study site, and the study was conducted according to the ethical principles of the Declaration of Helsinki. Although informed consent was not obtained, patients were given the opportunity to decline to have their clinical records used for research (opt-out consent provision). This study was registered at Clinicaltrials.gov (NCT03569436).

The study includes patients with recurrent or metastatic (distant sites) HNC cancer who were treated with nivolumab for the first time between July 1, 2017, and December 31, 2017. All eligible patients were included except those who had participated in a clinical trial with antineoplastic therapy. 
A

\begin{tabular}{llllclc}
\hline & $\mathbf{N}$ & ORR $(\mathbf{9 5 \%} \mathbf{C l}), \%$ & \multicolumn{4}{c}{ BOR, $\mathbf{n}(\%)$} \\
\cline { 4 - 7 } & & & $\mathbf{C R}$ & PR & SD & PD \\
\hline All cases & 223 & $15.7(11.2-21.1)$ & $3(1.3)$ & $32(14.3)$ & $61(27.4)$ & $127(57.0)$ \\
\hline
\end{tabular}

B

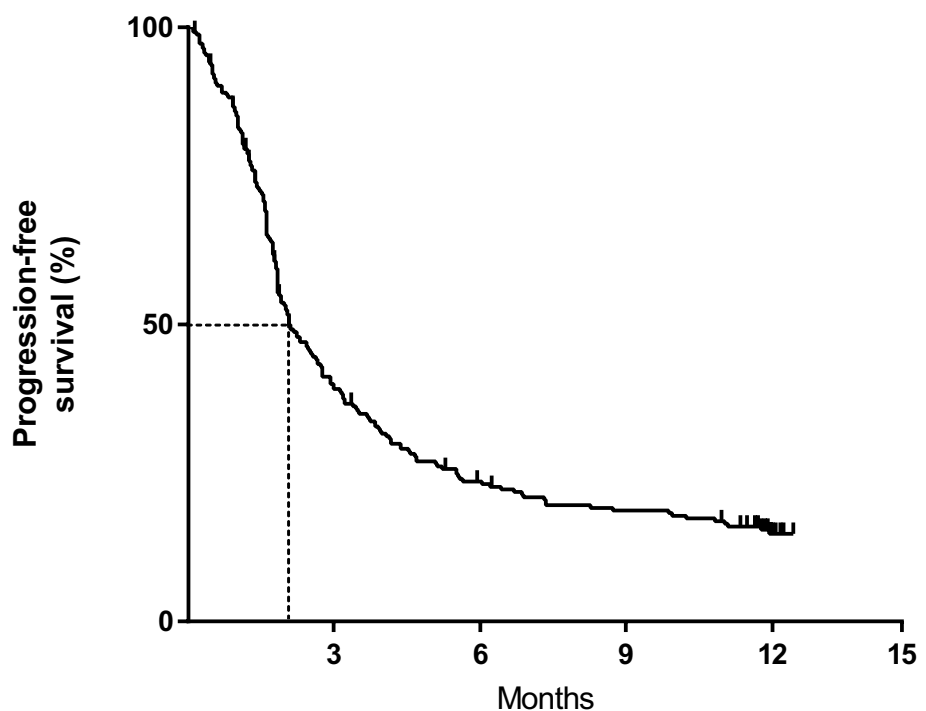

$\begin{array}{llllll}\text { No. at risk } & 256 & 94 & 54 & 42 & 17\end{array}$

\begin{tabular}{cccc}
\hline $\mathbf{N}$ & $\begin{array}{c}\text { Median (95\%Cl) } \\
\text { PFS (months) }\end{array}$ & $\begin{array}{c}\text { 6-month PFS rate } \\
\text { (\%) }\end{array}$ & $\begin{array}{c}\text { 12-month PFS rate } \\
\text { (\%) }\end{array}$ \\
\hline 256 & $2.1(1.8-2.7)$ & 23.6 & 14.8 \\
\hline
\end{tabular}

C

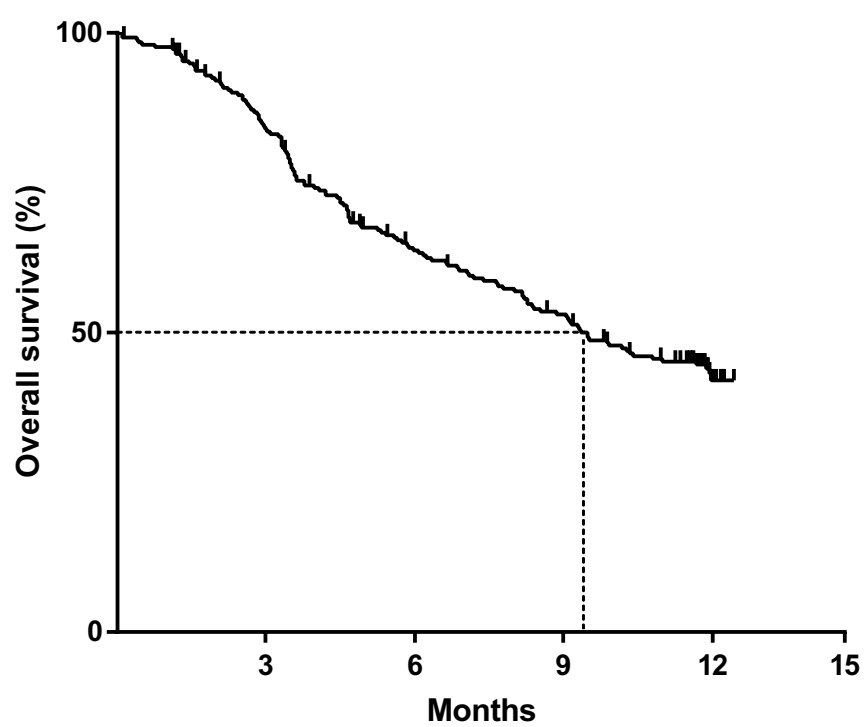

No. at risk

256

206

150

123

36

\begin{tabular}{cccc}
\hline $\mathbf{N}$ & $\begin{array}{c}\text { Median (95\%Cl) OS } \\
\text { (months) }\end{array}$ & $\begin{array}{c}\text { 6-month OS rate } \\
\text { (\%) }\end{array}$ & $\begin{array}{c}\text { 12-month OS rate } \\
\text { (\%) }\end{array}$ \\
\hline 256 & $9.5(8.2-12.0)$ & 63.7 & 43.2 \\
\hline
\end{tabular}




\begin{tabular}{|c|c|c|c|c|c|c|}
\hline \multirow[t]{2}{*}{ Primary site } & \multirow[t]{2}{*}{$\mathbf{N}$} & \multirow[t]{2}{*}{ ORR (95\% Cl), \% } & \multicolumn{4}{|c|}{ BOR, n (\%) } \\
\hline & & & CR & PR & SD & PD \\
\hline Nasopharynx & 19 & $21.1(6.1-45.6)$ & $0(0.0)$ & $4(21.1)$ & $9(47.4)$ & $6(31.6)$ \\
\hline Oropharynx & 35 & $8.6(1.8-23.1)$ & $0(0.0)$ & $3(8.6)$ & $10(28.6)$ & $22(62.9)$ \\
\hline Hypopharynx & 53 & $24.5(13.8-38.3)$ & $0(0.0)$ & $13(24.5)$ & $10(18.9)$ & $30(56.6)$ \\
\hline Oral cavity & 43 & $14.0(5.3-27.9)$ & $1(2.3)$ & $5(11.6)$ & $12(27.9)$ & $25(58.1)$ \\
\hline Maxillary sinus* & 14 & $7.1(0.2-33.9)$ & $1(7.1)$ & $0(0.0)$ & $1(7.1)$ & $12(85.7)$ \\
\hline Larynx & 19 & $15.8(3.4-39.6)$ & $0(0.0)$ & $3(15.8)$ & $5(26.3)$ & $11(57.9)$ \\
\hline Salivary gland* & 22 & $13.6(2.9-34.9)$ & $1(4.5)$ & $2(9.1)$ & $9(40.9)$ & $10(45.5)$ \\
\hline
\end{tabular}

B
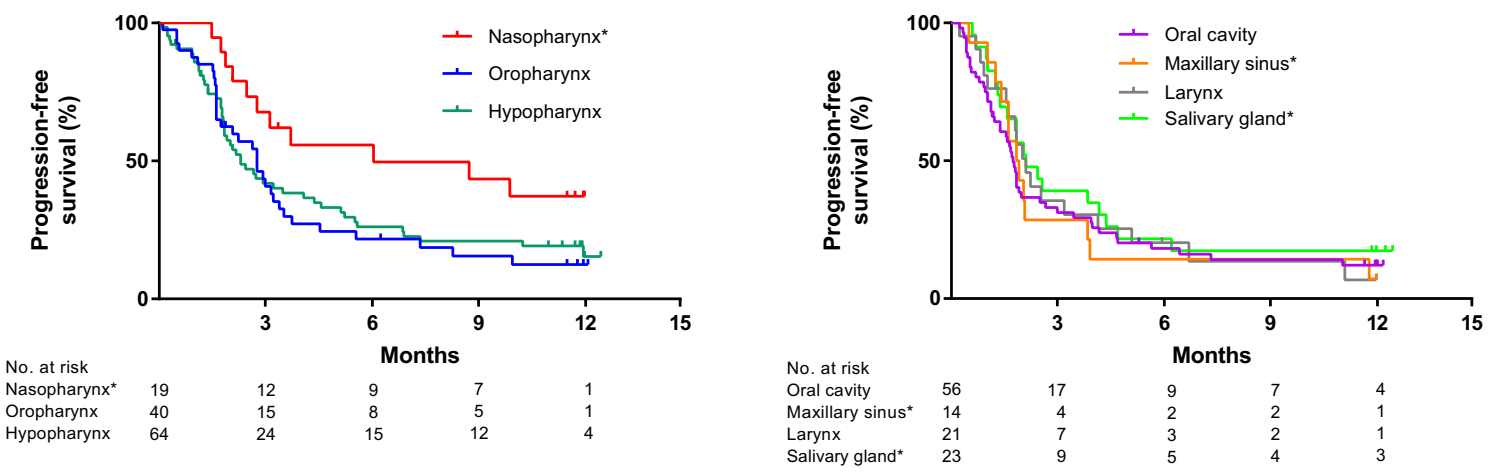

\begin{tabular}{|c|c|c|c|c|}
\hline Primary site & $\mathbf{N}$ & Median (95\% Cl) PFS (months) & 6-month PFS rate (\%) & 12-month PFS rate (\%) \\
\hline Nasopharyn $x^{*}$ & 19 & $6.1(2.5-\mathrm{NR})$ & 55.8 & 37.2 \\
\hline Oropharynx & 40 & $2.8(1.6-3.4)$ & 21.7 & 12.4 \\
\hline Hypopharynx & 64 & $2.3(1.8-4.1)$ & 26.2 & 15.3 \\
\hline Oral cavity & 56 & $1.7(1.4-2.0)$ & 18.2 & 12.1 \\
\hline Maxillary sinus* & 14 & $1.9(1.3-3.8)$ & 14.3 & 7.1 \\
\hline Larynx & 21 & $2.1(1.5-4.1)$ & 20.3 & 6.8 \\
\hline Salivary gland* & 23 & $2.1(1.4-4.2)$ & 21.7 & 17.4 \\
\hline
\end{tabular}

C

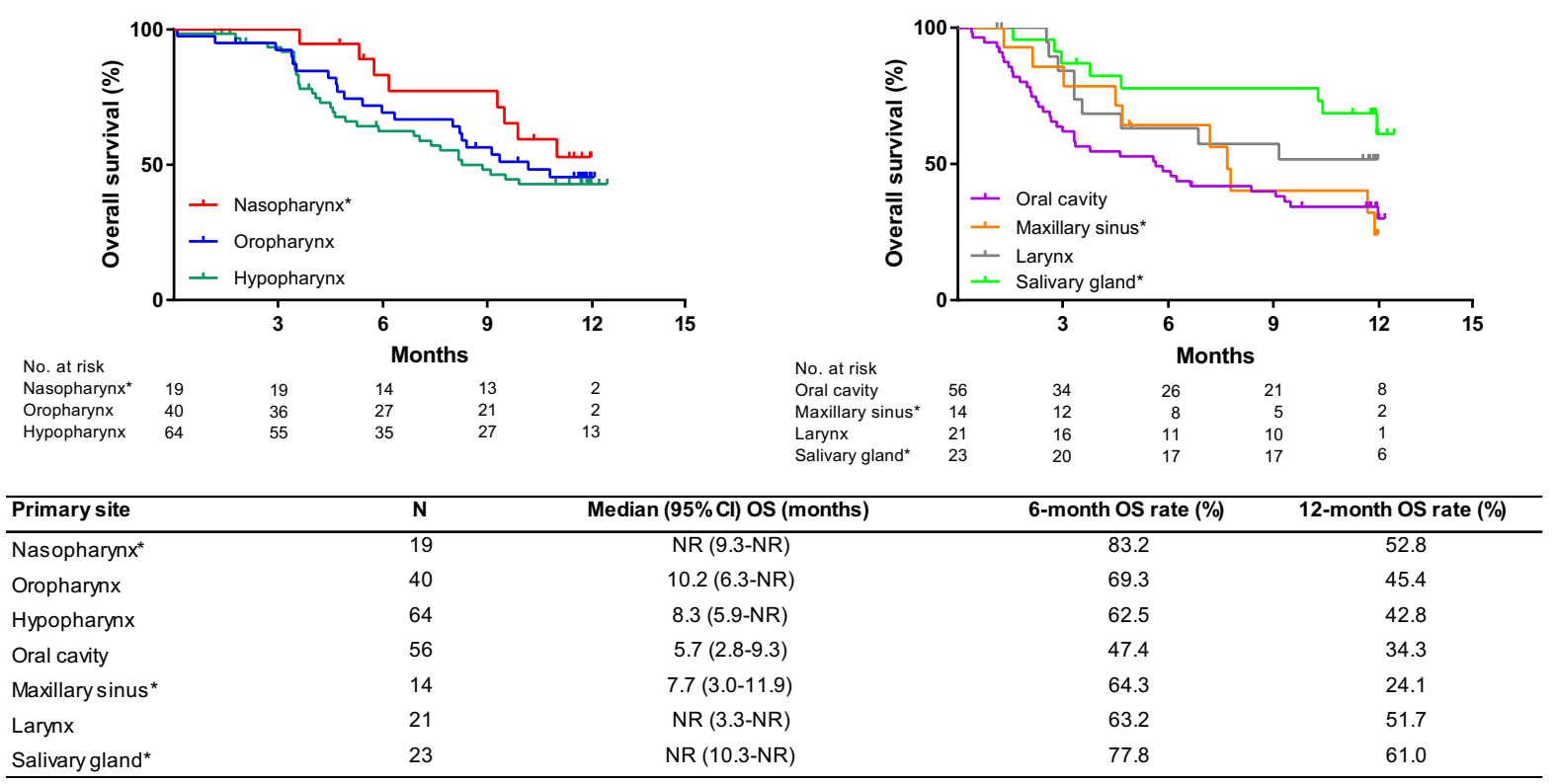


४Fig. 2 a Overall response rate b progression-free survival and c overall survival among patients stratified according to the primary site. $B O R$ best overall response, $C R$ complete response, $N R$ not reached, $O R R$ objective response rate, $O S$ overall survival, $P D$ progressive disease, $P F S$ progression-free survival, $P R$ partial response, $S D$ stable disease. *Primary tumor types excluded from Checkmate 141 study

The data were extracted from patients' medical charts into a specific electronic case report form. Data were collected from baseline until the most recent patient visit. The data cut-off date was 1 year after the first treatment of nivolumab in each patient. Baseline was defined as the visit prior to the start of nivolumab therapy, but the chart review encompassed the period from the diagnosis of HNC to collect data on therapies received prior to nivolumab.

\section{Endpoints}

The primary objectives were to determine the overall effectiveness, including BOR, progression-free survival (PFS), and OS, and to evaluate immune-related adverse events (AEs) in realworld clinical practice. Progression and response primarily recorded by physicians were assessed according to investigator-assessment Response Evaluation Criteria in Solid Tumors (RECIST) 1.1 criteria [6]. Evaluation time was not set due to the nature of this study.

AEs were classified according to the International Council for Harmonisation of Technical Requirements for Pharmaceuticals for Human Use Medical Dictionary for Regulatory Activities Japanese edition (MedDRA/J) Version 21.0.

Drug use information was collected by recording the doses of nivolumab that the patient received, the treatments received before and after administration of nivolumab and their outcomes, the duration of nivolumab treatment, the line of therapy in which patients received nivolumab, any changes in dose or dose interruptions made as a result of AEs, and eventual reason for discontinuation of nivolumab. Platinum-refractory disease in the context of primary therapy was defined as cancer progression within 6 months after the last administration of platinum [1]. Platinum-sensitive disease was defined as cancer progression from 6 months or longer after the last administration of platinum [1].

\section{Statistical analysis}

Effectiveness and safety analyses were performed with all patients who had received $\geq 1$ dose of nivolumab. Demographic and baseline characteristics, response data, and AEs were summarized using descriptive statistics (number of patients, mean and standard deviation) for continuous efficacy variables, and frequency and percentage for categorical variables. OS and PFS were estimated and plotted using the Kaplan-Meier method and expressed as the proportion of patients who survived to a specific point in time and median duration, with the corresponding two-sided $95 \%$ confidence intervals (CI). For subgroup analyses, tests of statistical significance were conducted using the log-rank test.

Statistical analyses were conducted using SAS Version 9.4 (SAS Institute, Japan).

\section{Results}

\section{Patient disposition and characteristics}

Among 256 registered patients, $79 \%$ were men and the median age was 66 years (range $20-84$ years; Table 1). Of the 246 patients with known ECOG PS score, 31 patients (12.6\%) had a performance status of $\geq 2$. Overall, 198 of the 239 patients $(82.8 \%)$ with known disease stage had stage III or IV disease at the time of HNC diagnosis (Table 1). Most patients $(n=217 ; 84.8 \%)$ had squamous cell carcinoma, but 29 patients $(11.3 \%)$ had non-squamous cell histology. Primary tumor sites were the hypopharynx $(n=64 ; 25.0 \%)$, oral cavity $(n=56 ; 21.9 \%)$, oropharynx $(n=40 ; 15.6 \%)$, salivary gland $(n=23 ; 9.0 \%)$, larynx $(n=21 ; 8.2 \%)$, nasopharynx $(n=19 ; 7.4 \%)$, maxillary sinus $(n=14 ; 5.5 \%)$, and other sites $(n=19 ; 7.4 \%)$.

Seventy patients $(27.3 \%)$ received nivolumab as the firstline treatment for recurrent/metastatic HNC, $110(43.0 \%)$ as second-line, and $76(29.7 \%)$ as third-line or later treatment.

After the 12-month follow-up, 14.1\% $(n=36)$ were still under the treatment with nivolumab. Of the 220 patients (85.9\%) who completed nivolumab treatment, 170 patients (77.3\%) had shown disease progression and nine patients had died. The median ( $\min , \max$ ) duration of nivolumab treatment was $72.5(1,380)$ days, with patients receiving a median of 6.0 (range 1-27) doses of nivolumab (Table S1).

\section{Overall effectiveness}

The BOR was assessed in 223 of 256 evaluable patients. Among these 223 patients, the BOR was CR in 3 patients $(1.3 \%)$ and PR in 32 patients $(14.3 \%)$, for an objective response rate (ORR) of 15.7 (95\% CI 11.2-21.1) \% $(n=35)$. An additional 61 patients $(27.4 \%)$ had SD, resulting in a disease control rate of $43.0 \%$ (Fig. 1a). The median duration of response was 5.1 (95\% CI 2.8-NE) months. The median PFS was 2.1 (95\% CI 1.8-2.7) months (Fig. 1b) and the median OS in the 256 patients treated with nivolumab was $9.5(95 \%$ CI 8.2-12.0) months (Fig. 1c). The estimated 12-month OS rate was 43.2 (95\% CI 36.7-49.5)\%, with an estimated 12-month PFS rate of 14.8 (95\% CI 10.5-19.7)\%. 


\section{A}

\begin{tabular}{lcccccc}
\hline Platinum sensitivity & N & ORR (95\% Cl), \% & \multicolumn{4}{c}{ BOR, $\mathbf{n}(\%)$} \\
\cline { 3 - 6 } & & & CR & PR & SD & PD \\
\hline Sensitive & 31 & $16.1(5.5-33.7)$ & $1(3.2)$ & $4(12.9)$ & $8(25.8)$ & $18(58.1)$ \\
Refractory & 130 & $16.2(10.3-23.6)$ & $1(0.8)$ & $20(15.4)$ & $30(23.1)$ & $79(60.8)$ \\
\hline
\end{tabular}

B

No. at risk Pt-sensitive Pt-refractory

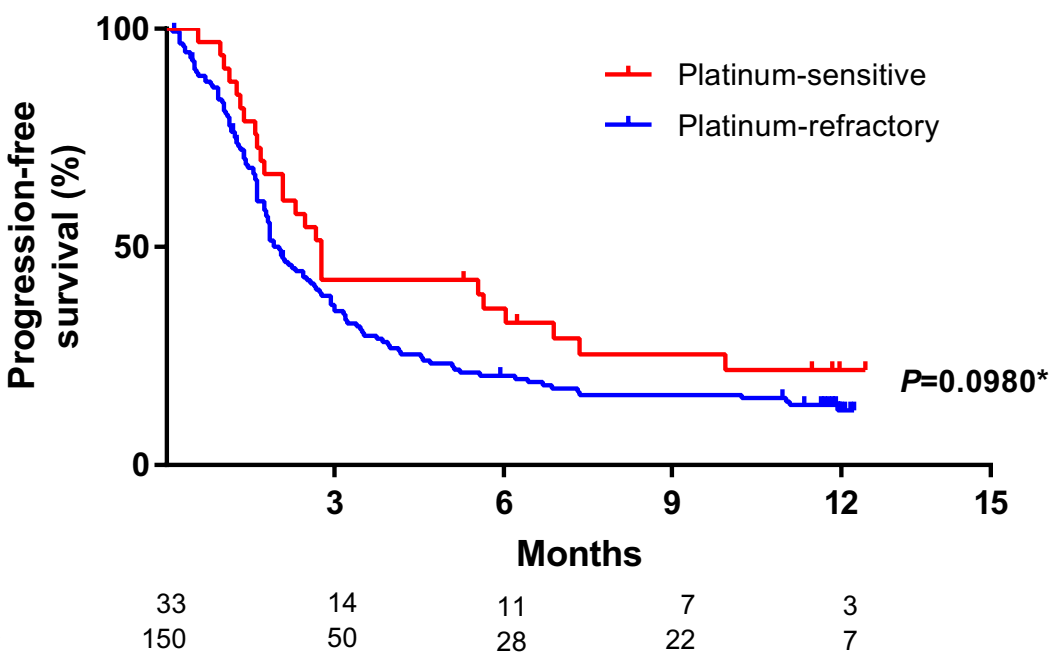

\begin{tabular}{lcccc}
\hline $\begin{array}{l}\text { Platinum } \\
\text { sensitivity }\end{array}$ & N & $\begin{array}{c}\text { Median (95\% Cl) } \\
\text { PFS (months) }\end{array}$ & $\begin{array}{c}\text { 6-month PFS } \\
\text { rate (\%) }\end{array}$ & $\begin{array}{c}\text { 12-month PFS } \\
\text { rate (\%) }\end{array}$ \\
\hline Sensitive & 33 & $2.8(1.7-6.1)$ & 35.9 & 21.8 \\
Refractory & 150 & $2.0(1.7-2.6)$ & 20.5 & 12.6 \\
\hline
\end{tabular}

C

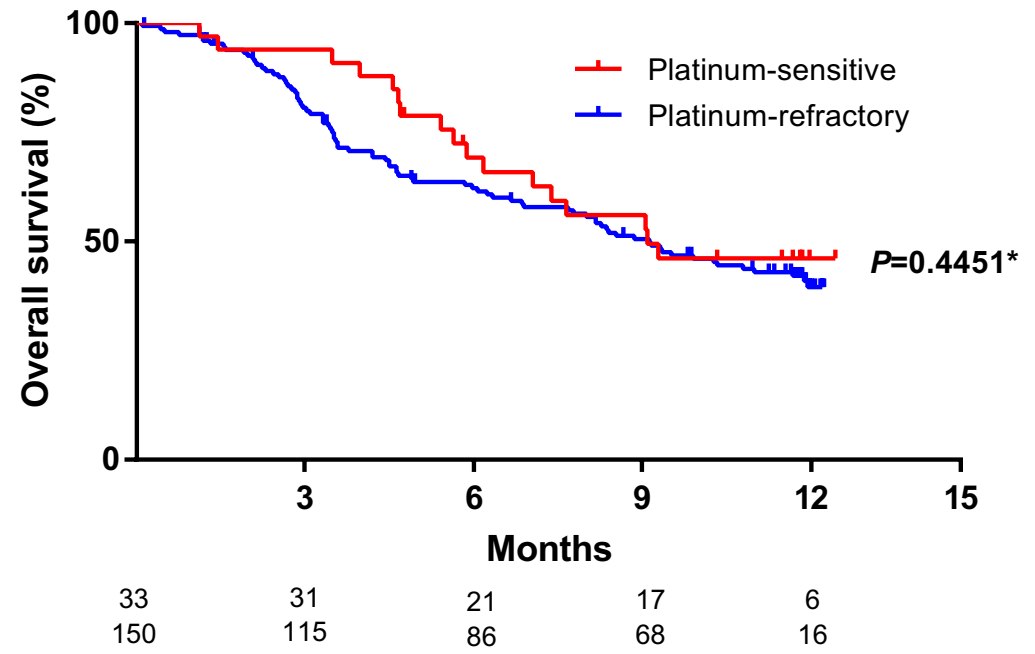

No. at risk

Pt-sensitive

Pt-refractory
150
21
86

68
16

\begin{tabular}{lcccc}
\hline $\begin{array}{l}\text { Platinum } \\
\text { sensitivity }\end{array}$ & N & $\begin{array}{c}\text { Median (95\% Cl) } \\
\text { OS (months) }\end{array}$ & $\begin{array}{c}\text { 6-month OS } \\
\text { rate (\%) }\end{array}$ & $\begin{array}{c}\text { 12-month OS } \\
\text { rate (\%) }\end{array}$ \\
\hline Sensitive & 33 & $9.1(6.2-\mathrm{NR})$ & 69.2 & 46.1 \\
Refractory & 150 & $9.1(6.9-11.9)$ & 62.2 & 39.5 \\
\hline
\end{tabular}


4Fig. 3 a Overall response rate b progression-free survival and $\mathbf{c}$ overall survival among patients stratified according to platinum-sensitive or platinum-refractory status. BOR best overall response, $C R$ complete response, $O R R$ objective response rate, $O S$ overall survival, $P D$ progressive disease, $P F S$ progression-free survival, $P R$ partial response, $S D$ stable disease. ${ }^{*}$ Log-rank test

\section{Effectiveness by subgroup}

Regarding the effectiveness of nivolumab in Japanese patients with primary tumor sites specifically excluded in the CheckMate 141 study, the ORR was 21.1 (95\% CI 6.1-45.6) \%, 13.6 (95\% CI 2.9-34.9) \%, and 7.1 (95\% CI 0.2-33.9) \% in the nasopharynx, salivary glands, and maxillary sinus, respectively (Fig. 2a). The median PFS was 6.1 (95\% CI 2.5-NE) months in the nasopharynx, 2.1 (95\% CI 1.4-4.2) months in the salivary glands, and 1.9 (95\% CI 1.3-3.8) months in the maxillary sinus with 12-month PFS rates of $37.2 \%, 17.4 \%$, and $7.1 \%$ for the nasopharynx, salivary glands, and maxillary sinus, respectively (Fig. 2b). Finally, the median OS was not reached for the nasopharynx and salivary glands and was 7.7 (95\% CI 3.0-11.9) months in the maxillary sinus (Fig. 2c). Further, the 12-month OS rate in the nasopharynx, salivary glands, and maxillary sinus was $52.8 \%, 61.0 \%$, and $24.1 \%$, respectively. This study included a small number of patients $(n=29)$ with non-squamous cell carcinoma (non-SCC) in addition to patients with SCC $(n=217)$. Effectiveness according to histological type (SCC versus non-SCC) is shown in Figure S1. ORR, PFS, and OS were similar between patients with SCC or non-SCC with no statistically significant differences noted for any comparison. The primary site and histology of patients with non-SCC are detailed in Table $\mathrm{S} 2$.

In the present real-world study, nivolumab use was not limited to platinum-refractory but also platinum-sensitive patients. In a subgroup analysis by prior platinum responsiveness, the ORR was 16.2 (95\% CI 10.3-23.6)\% and 16.1 (95\% CI 5.5-33.7) \% in platinum-refractory patients and platinum-sensitive patients, respectively (Fig. 3a). The median PFS was 2.0 (95\% CI 1.7-2.6) months in platinumrefractory and 2.8 (95\% CI 1.7-6.1) months in platinum-sensitive (Fig. 3b). The median OS was 9.1 (95\% CI 6.9-11.9) months and 9.1 (95\% CI 6.2-NR) months for platinumrefractory and platinum-sensitive patients, respectively (Fig. 3c).

In a subgroup analysis by PS, the ORR, PFS, and OS were generally numerically greater in patients with ECOG PS 0 status, compared with patients with ECOG PS 1 or, in particular, ECOG PS $\geq 2$ status (Fig. 4). The ORR was 17.9 (95\% CI 11.3-26.2) \%, 14.1 (95\% CI 7.5-23.4) \%, and 5.6 (95\% CI 0.1-27.3) \% in patients with ECOG PS 0, ECOG PS 1, and ECOG PS $\geq 2$ status, respectively (Fig. 4a). PFS was 2.6 (95\% CI 2.0-3.8) months, 2.1 (95\% CI 1.7-2.8) months, and 1.4 (95\% CI 0.8-2.2) months in patients with ECOG PS 0 , ECOG PS 1 , and ECOG PS $\geq 2$ status, respectively (Fig. 4b). The median OS was not reached in patients with ECOG PS 0 status and was 6.9 (95\% CI 4.9-10.4) months and 3.1 (95\% CI 2.1-3.6) months in patients with ECOG PS 1 and ECOG PS $\geq 2$ status, respectively (Fig. 4c).

Effectiveness by BOR at 3 months are shown in Fig. 5. Both PFS and OS were greater in patients with CR/PR or SD compared with patients who experienced progressive disease (Fig. 5a, b). Median PFS was not reached in patients with CR/PR and was 4.7 (95\% CI 4.1-6.7) months and 1.6 (95\% CI 1.4-1.6) months in patients with SD and PD, respectively (Fig. 5a). Median OS was not reached in patients with SD or CR/PR at 3 months and 6.2 (95\% CI 4.6-7.8) months in patients with progressive disease (Fig. 5b).

\section{Incidence of AEs}

Thirty-eight patients (14.8\%) developed immune-related AEs of any grade; 15 patients $(5.9 \%)$ developed a grade $\geq 3$ immune-related AE (Table 2). Endocrine disorders were the most common type of immune-related AEs which affected 14 patients, but only two of these events were grade $\geq 3$. The most common type of grade $\geq 3$ immune-related AEs were lung disorders $(n=6)$. One patient developed a grade 5 immune-related AE (interstitial pneumonia). The median time to onset of any immune-related AEs was 8.7 (0.1-43.7) weeks; each categorized immune-related AE widely appeared throughout the 12-month observation periods. Median time to resolution of most immune-related AEs was 3 to 6 weeks.

\section{Discussion}

To the best of our knowledge, this is the largest study to evaluate the effectiveness and safety of nivolumab in patients with HNC in Japanese real-world clinical practice. Median PFS (2.1 vs 2.0 months), 12-month OS rate (43\% vs $36 \%$ ), and median OS (9.5 vs 7.5 months) in our study were similar to those in CheckMate 141 [1]. Furthermore, our data are also comparable to other reported real-world data in Japan $[7,8]$.

The current study addresses several data gaps between the clinical trial and real-world settings. First of all, the current study reveals the effectiveness of nivolumab in patients with primary tumors in the nasopharynx, maxillary sinus, or salivary gland, who would have been excluded from previous phase III clinical trials for HNC including the CheckMate 141, KEYNOTE-040, KEYNOTE-048, and EXTREME studies [9-11]. In particular, we found a patient with maxillary sinus cancer responded to nivolumab, which is the first positive result recorded for 
A

\begin{tabular}{lcccccc}
\hline ECOG status & $\mathbf{N}$ & ORR (95\% Cl), \% & \multicolumn{4}{c}{ BOR, $\mathbf{n}$ (\%) } \\
\cline { 4 - 6 } & & & $\mathbf{C R}$ & $\mathbf{P R}$ & $\mathbf{S D}$ & $\mathbf{P D}$ \\
\hline 0 & 112 & $17.9(11.3-26.2)$ & $1(0.9)$ & $19(17.0)$ & $36(32.1)$ & $56(50.0)$ \\
1 & 85 & $14.1(7.5-23.4)$ & $2(2.4)$ & $10(11.8)$ & $20(23.5)$ & $53(62.4)$ \\
$\geq 2$ & 18 & $5.6(0.1-27.3)$ & $0(0.0)$ & $1(5.6)$ & $3(16.7)$ & $14(77.8)$ \\
\hline
\end{tabular}

B

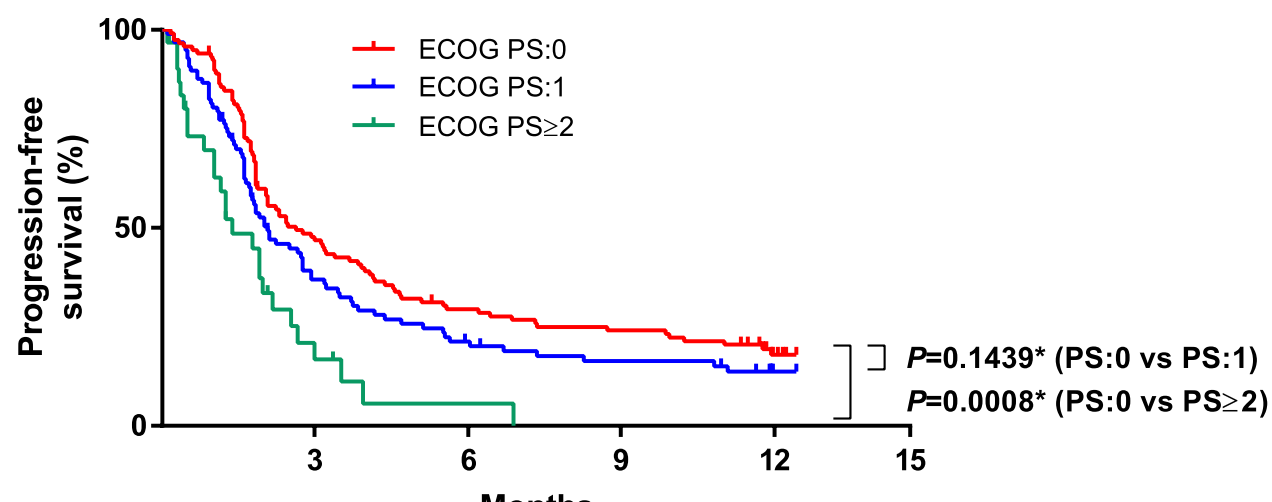

\begin{tabular}{|c|c|c|c|c|c|}
\hline \multicolumn{2}{|l|}{ No. at risk } & \multicolumn{3}{|c|}{ Months } & \\
\hline ECOG PS:0 & 118 & 54 & 33 & 27 & \\
\hline ECOG PS: 1 & 97 & 33 & 18 & 13 & \\
\hline$E C O G$ PS $\geq 2$ & 31 & 4 & 1 & 0 & \\
\hline \multicolumn{2}{|c|}{$\overline{\text { ECOG status }}$} & $\mathbf{N}$ & $\begin{array}{c}\text { Median }(95 \% \mathrm{Cl}) \\
\text { PFS (months) }\end{array}$ & $\begin{array}{c}\text { 6-month PFS } \\
\text { rate }(\%)\end{array}$ & $\begin{array}{c}\text { 12-month PFS } \\
\text { rate }(\%)\end{array}$ \\
\hline \multicolumn{2}{|l|}{0} & 118 & $2.6(2.0-3.8)$ & 29.4 & 18.0 \\
\hline \multicolumn{2}{|l|}{1} & 97 & $2.1(1.7-2.8)$ & 21.3 & 13.7 \\
\hline \multicolumn{2}{|c|}{$\geq 2$} & 31 & $1.4(0.8-2.2)$ & 5.6 & 0.0 \\
\hline
\end{tabular}

C

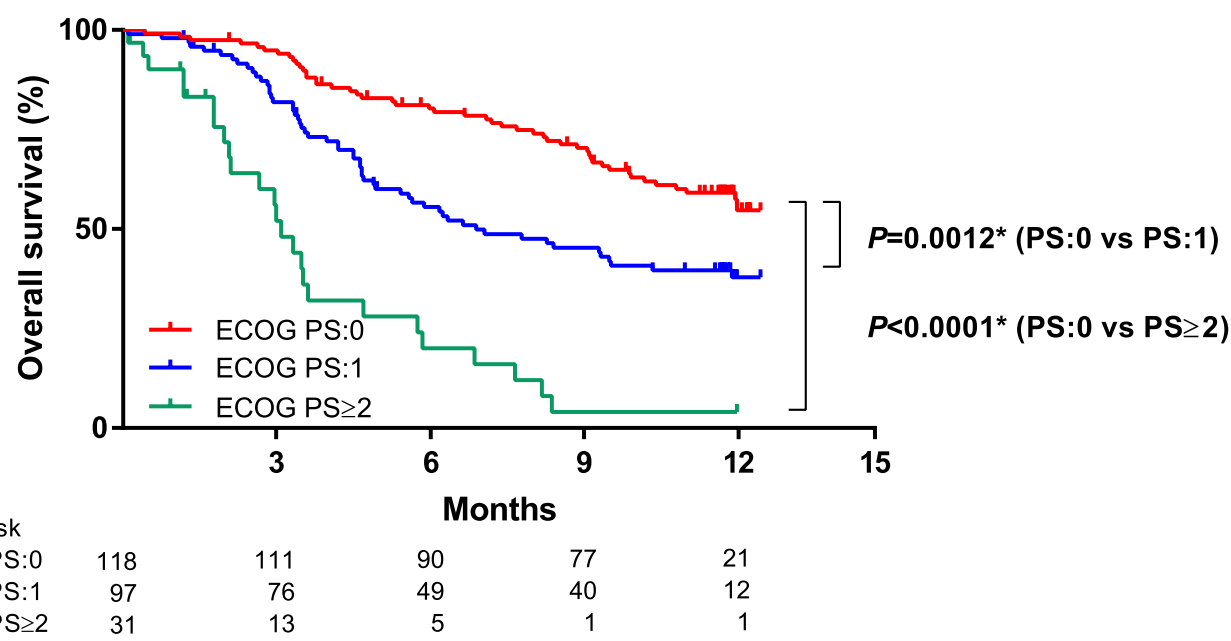

\begin{tabular}{lcccc}
\hline ECOG status & $\mathbf{N}$ & $\begin{array}{c}\text { Median (95\%Cl) } \\
\text { OS (months) }\end{array}$ & $\begin{array}{c}\text { 6-month OS } \\
\text { rate (\%) }\end{array}$ & $\begin{array}{c}\text { 12-month OS } \\
\text { rate (\%) }\end{array}$ \\
\hline 0 & 118 & NR (11.0-NR) & 80.3 & 57.5 \\
1 & 97 & $6.9(4.9-10.4)$ & 55.5 & 37.8 \\
$\geq 2$ & 31 & $3.1(2.1-3.6)$ & 20.0 & 4.0 \\
\hline
\end{tabular}


४Fig. 4 a Overall response rate b progression-free survival and $\mathbf{c}$ overall survival among patients according to ECOG status. BOR best overall response, $C R$ complete response, ECOG PS Eastern Cooperative Oncology Group performance status, $N R$ not reached, $O R R$ objective response rate, $O S$ overall survival, $P D$ progressive disease, $P F S$ progression-free survival, $P R$ partial response, $S D$ stable disease. *Log-rank test

any checkpoint inhibitor monotherapies in such patients [12]. In addition, the effectiveness of nivolumab for nasopharyngeal and salivary gland tumors in the current study was consistent with the results in prior phase I/II trials [9, 13-15]. Historical data showed that prognosis is generally poor in patients with primary HNC in the hypopharynx, oral cavity, and nasal sinuses, whereas prognosis tends towards improvement in patients with nasopharyngeal or laryngeal primary tumors [10, 16-19]. It has been suggested that the primary $\mathrm{HNC}$ site is associated with poor response to chemotherapy [20]. By contrast, the current study showed that patients well responded to nivolumab irrespective of the expected prognosis.
Fig. 5 a Progression-free survival and $\mathbf{b}$ overall survival according to the best overall response at 3 months. $C R$ complete response, $O S$ overall survival, $P D$ progressive disease, $P F S$ progression-free survival, $P R$ partial response, $S D$ stable disease. *Log-rank test

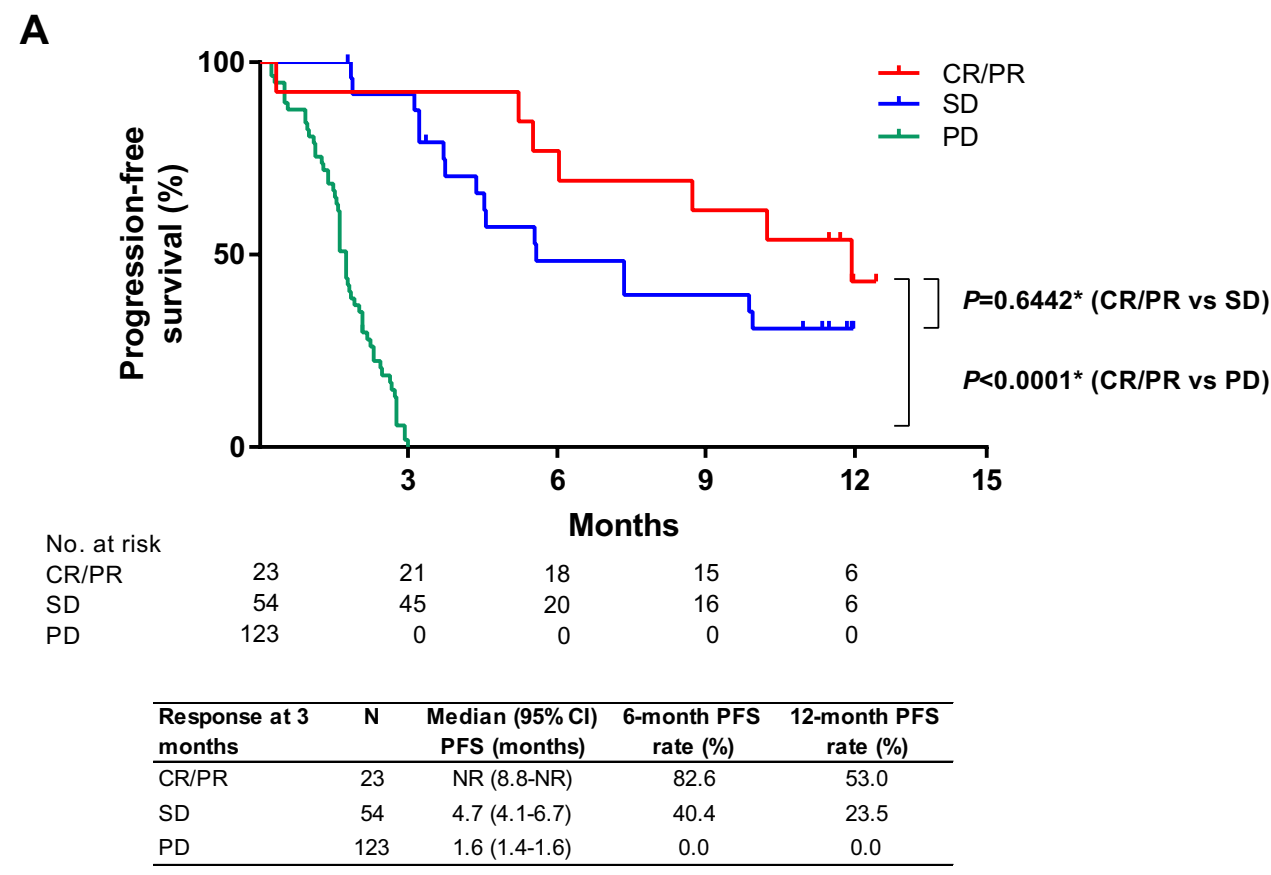

B

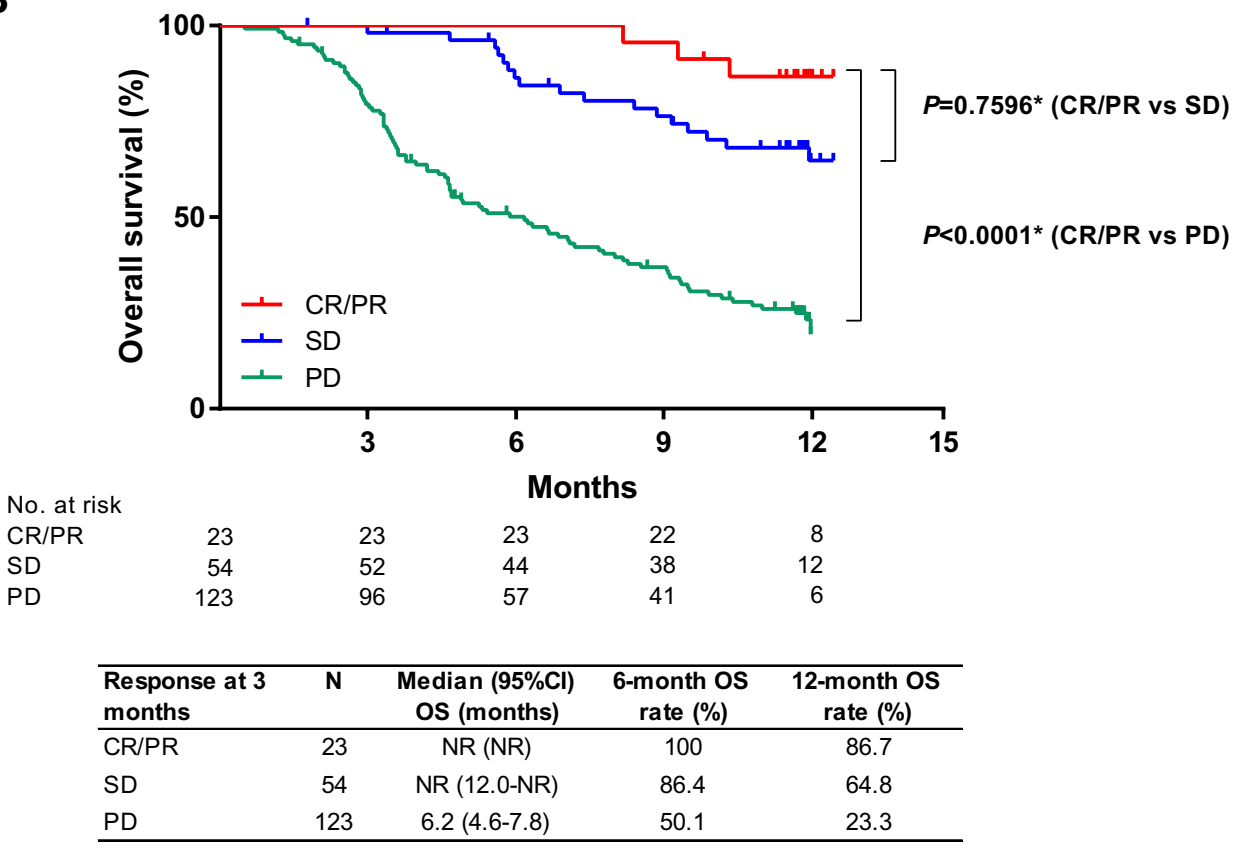


Table 2 Incidence of immune-related adverse events over 12 months

\begin{tabular}{llllllll}
\hline Immune-related AE category & \multicolumn{2}{l}{ No. of patients, $n(\%)$} & & No. of events & \\
\cline { 2 - 3 } & Any grade & Grade $\geq 3$ & & All grade, $n$ & $\begin{array}{l}\text { Median (range) time } \\
\text { to onset, weeks }\end{array}$ & $\begin{array}{l}\text { Resolved, } n \\
\text { (recovery rate, \%) }\end{array}$ & $\begin{array}{l}\text { Median (range) time } \\
\text { to resolution, weeks }\end{array}$ \\
\hline Any immune-related AE & $38(14.8)$ & $15(5.9)$ & 47 & $8.7(0.1-43.7)$ & $34(72.3)$ & $4.8(0-43.4)$ \\
Endocrine disorder & $14(5.5)$ & $2(0.8)$ & 14 & $9.3(0.1-43.0)$ & $9(64.3)$ & $5.1(1.4-12.0)$ \\
Lung disorder & $8(3.1)$ & $6(2.3)$ & 8 & $3.0(0.3-27.3)$ & $4(50.0)$ & $4.8(3.0-11.3)$ \\
Skin disorder & $7(2.7)$ & $2(0.8)$ & 8 & $21.2(4.0-33.1)$ & $6(75.0)$ & $2.5(2.0-22.0)$ \\
Liver disorder & $6(2.3)$ & $3(1.2)$ & 7 & $12.0(2.0-29.0)$ & $7(100.0)$ & $4.7(0.7-27.3)$ \\
Gastrointestinal disorder & $3(1.2)$ & $1(0.4)$ & 3 & $9.4(9.0-43.7)$ & $3(100.0)$ & $6.4(0-14.0)$ \\
Blood disorder & $2(0.8)$ & $1(0.4)$ & 2 & $5.8(4.0-7.6)$ & $1(50.0)$ & $43.4(43.4-43.4)$ \\
Other & $5(2.0)$ & $2(0.8)$ & 5 & $4.0(2.0-29.0)$ & $4(80.0)$ & $3.1(0-27.0)$ \\
\hline
\end{tabular}

Time to onset refers to the time after the first dose of nivolumab and time to resolution refers to time from the onset of the adverse event until complete resolution

$A E$ adverse event

The effectiveness of nivolumab for platinum-refractory HNC in this study was comparable to that of CheckMate 141. While most patients receiving nivolumab in real-world clinical practice have platinum-refractory disease, $18 \%$ of the patients in our study had platinum-sensitive disease, a population that was not included in CheckMate 141 [1]. In the present study, ORR and OS were numerically similar between patient populations with platinum-refractory and platinum-sensitive diseases. However, because the effectiveness of the platinum-sensitive disease has been evaluated only in a limited number of patients [9], further large-scale studies are warranted. Stratification of patients by performance status in this study showed that nivolumab was most effective, in terms of ORR, PFS, and OS, in patients with ECOG PS 0 status. Other studies utilizing anti-PD-1 inhibitors, including nivolumab, in various real-world settings including advanced melanoma, non-small cell lung cancer, and head and neck cancer have demonstrated that favorable PS is predictive of greater OS and PFS [21-23]. Recent longitudinal studies of anti-PD-1 inhibitors, also conducted in real-world clinical practice settings with median follow-up periods of up to 12.9 months, further verify better responses in patients with ECOG PS 0-1 status versus those with ECOG PS $>1$ status [24-26]. Therefore, the present results provide additional evidence to support the notion that nivolumab and similar agents in this class are more effective in patients who are in good general condition at treatment initiation.

Superior PFS and OS were observed in patients whose BOR at 3 months was CR, PR, or SD. This is consistent with previous findings with nivolumab in patients with melanoma or non-small cell lung cancer $[4,5]$, and is also consistent with the known response profile to immune-checkpoint inhibitors. In addition, even after tumor progression, half of the patients with PD survived for more than 6 months. These patients may receive subsequent chemotherapy after discontinuation of the nivolumab therapy, which may contribute to prolonged survival because it has been reported for several cancers that chemotherapy after immunotherapy is highly effective [27-30]. Future studies with longer follow-up will reveal possibly different impacts of subsequent chemotherapy.

The incidence and timing of immune-related AEs in our study were similar to or even lower than previous reports, including CheckMate 141 [1,31], and with Japanese clinical use of nivolumab in patients with HNC [8]. No new safety signals were identified in our study compared with CheckMate 141, despite the fact that our study included a more vulnerable patient population. These data indicate that nivolumab has a well-characterized and manageable safety profile in real-world clinical use.

Retrospective observational studies have limitations. As an observational design, there was no control group. Because completed by individual physicians during realworld clinical practice, patients' medical records may not always contain complete and comparable information and may contain measurement errors. To recruit a large number of patients, we preferentially included centers that treat a high number of patients with HNC. This may have introduced some selection bias in the study population.

In conclusion, this retrospective observational analysis in a real-world clinical setting supports the effectiveness and safety of nivolumab in a range of Japanese patients with HNC. No new safety signals were identified, and the findings of randomized clinical trials with nivolumab are applicable to a real-world population of patients with a more diverse clinical profile in terms of performance status and primary site. 
Acknowledgements The authors thank all clinicians for their involvement and contribution to the study. The data for this study were collected and analyzed by Mebix, Inc., Tokyo, Japan. The authors also thank Yoshiko Okamoto, Ph.D. and Catherine Rees, of inScience Communications, Springer Healthcare, for writing the outline and the first draft of the manuscript. This medical writing assistance was funded by Bristol-Myers Squibb and Ono Pharmaceutical.

Author contributions AH, YS, ID, and TY contributed to the study conception and design. NH, YS, SK, RY, TY, TF, KT, MY, KH, TU, HH, ST, TO, DS, MY, AW, KO, TY, NM, NK, MA, DS, TA, and AH were involved in data acquisition. $\mathrm{NH}$, ID, and TY performed data analysis and interpretation. The first draft of the manuscript was written by $\mathrm{NH}$, and TY. All authors reviewed and commented on subsequent drafts of the manuscript. All authors read and approved the final manuscript. All named authors meet the International Committee of Medical Journal Editors (ICMJE) criteria for authorship for this article, take responsibility for the integrity of the work as a whole, and have given their approval for this version to be published.

Funding This study was funded by Bristol-Myers Squibb and Ono Pharmaceutical.

\section{Compliance with ethical standards}

Conflict of interest N. Hanai, Y. Shimizu, S. Kariya, R. Yasumatsu, T. Fujii, M. Yoshida, K. Hanyu, H. Hirakawa, T. Ono, D. Sano, M. Yamauchi, K. Omori, N. Kudo, M. Arai, D. Sakurai, T. Asakage, A. Homma, T. Yokota, K. Tsukahara, S. Takahashi, and T. Yamazaki report grants from Bristol-Myers Squibb K.K. and Ono Pharmaceutical during the conduct of the study; K. Tsukahara, and S. Takahashi report personal fees from Bristol-Myers Squibb K.K. and Ono Pharmaceutical during the conduct of the study; T. Ueda reports grants from Ono Pharmaceutical; A. Watanabe, and N. Monden report nothing to disclose during the conduct of the study; I. Doi is an employee of Ono Pharmaceutical; T. Yamada is an employee of Bristol-Myers Squibb.

Ethical approval The study protocol was approved by the Institutional Review Board/Independent Ethics Committee at each study site. Informed consent was not obtained but patients were given the opportunity to decline to have their clinical records used for research.

Open Access This article is licensed under a Creative Commons Attribution 4.0 International License, which permits use, sharing, adaptation, distribution and reproduction in any medium or format, as long as you give appropriate credit to the original author(s) and the source, provide a link to the Creative Commons licence, and indicate if changes were made. The images or other third party material in this article are included in the article's Creative Commons licence, unless indicated otherwise in a credit line to the material. If material is not included in the article's Creative Commons licence and your intended use is not permitted by statutory regulation or exceeds the permitted use, you will need to obtain permission directly from the copyright holder. To view a copy of this licence, visit http://creativecommons.org/licenses/by/4.0/.

\section{References}

1. Ferris RL, Blumenschein G Jr, Fayette J et al (2016) Nivolumab for recurrent squamous-cell carcinoma of the head and neck. $\mathrm{N}$ Engl J Med 375(19):1856-1867

2. Ferris RL, Blumenschein G Jr, Fayette J et al (2018) Nivolumab vs investigator's choice in recurrent or metastatic squamous cell carcinoma of the head and neck: 2-year long-term survival update of CheckMate 141 with analyses by tumor PD-L1 expression. Oral Oncol 81:45-51

3. Kim ES, Bruinooge SS, Roberts S et al (2017) Broadening eligibility criteria to make clinical trials more representative: American Society of Clinical Oncology and Friends of Cancer Research Joint Research Statement. J Clin Oncol 35(33):3737-3744

4. Antonia SJ, Borghaei H, Ramalingam SS et al (2019) Four-year survival with nivolumab in patients with previously treated advanced non-small-cell lung cancer: a pooled analysis. Lancet Oncol 20(10):1395-1408

5. Yamazaki N, Kiyohara Y, Uhara H et al (2019) Long-term follow up of nivolumab in previously untreated Japanese patients with advanced or recurrent malignant melanoma. Cancer Sci 110(6):1995-2003

6. Eisenhauer EA, Therasse P, Bogaerts J et al (2009) New response evaluation criteria in solid tumours: revised RECIST guideline (version 1.1). Eur J Cancer 45(2):228-247

7. Hori R, Shinohara S, Kojima T et al (2019) Real-world outcomes and prognostic factors in patients receiving nivolumab therapy for recurrent or metastatic head and neck carcinoma. Cancers (Basel) 11(9): 1317

8. Okamoto I, Sato H, Kondo T et al (2019) Efficacy and safety of nivolumab in 100 patients with recurrent or metastatic head and neck cancer-a retrospective multicentre study. Acta Otolaryngol 139(10):918-925

9. Cohen EEW, Soulieres D, Le Tourneau C et al (2019) Pembrolizumab versus methotrexate, docetaxel, or cetuximab for recurrent or metastatic head-and-neck squamous cell carcinoma (KEYNOTE-040): a randomised, open-label, phase 3 study. Lancet 393(10167): 156-167

10. Vermorken JB, Mesia R, Rivera F et al (2008) Platinum-based chemotherapy plus cetuximab in head and neck cancer. $\mathrm{N}$ Engl J Med 359(11):1116-1127

11. Burtness B, Harrington KJ, Greil R et al (2019) Pembrolizumab alone or with chemotherapy versus cetuximab with chemotherapy for recurrent or metastatic squamous cell carcinoma of the head and neck (KEYNOTE-048): a randomised, open-label, phase 3 study. Lancet 394(10212):1915-1928

12. Borcoman E, Kanjanapan Y, Champiat S et al (2019) Novel patterns of response under immunotherapy. Ann Oncol 30(3):385-396

13. Ma BBY, Lim WT, Goh BC et al (2018) Antitumor activity of nivolumab in recurrent and metastatic nasopharyngeal carcinoma: an international, multicenter study of the Mayo Clinic Phase 2 Consortium (NCI-9742). J Clin Oncol 36(14):1412-1418

14. Ma Y, Fang W, Zhang Y et al (2019) A phase I/II open-label study of nivolumab in previously treated advanced or recurrent nasopharyngeal carcinoma and other solid tumors. Oncologist 24(7):891-e431

15. Rodriguez CP, Wu QV, Voutsinas J et al (2019) A phase II trial of pembrolizumab and vorinostat in recurrent metastatic head and neck squamous cell carcinomas and salivary gland cancer. Clin Cancer Res 26:837-845

16. Cooper JS, Porter K, Mallin K et al (2009) National Cancer Database report on cancer of the head and neck: 10-year update. Head Neck 31(6):748-758

17. Schneider K, Marbaix E, Bouzin C et al (2018) Immune cell infiltration in head and neck squamous cell carcinoma and patient outcome: a retrospective study. Acta Oncol 57(9):1165-1172

18. Talani C, Makitie A, Beran M et al (2019) Early mortality after diagnosis of cancer of the head and neck: a population-based nationwide study. PLoS ONE 14(10):e0223154

19. Japanese Society of Clinical Oncology (2015) New clinical oncology, 4th edn. Nankodo, Tokyo 
20. Depenni R, Cossu Rocca M, Ferrari D et al (2019) Clinical outcomes and prognostic factors in recurrent and/or metastatic head and neck cancer patients treated with chemotherapy plus cetuximab as first-line therapy in a real-world setting. Eur J Cancer 115:4-12

21. Morita R, Okishio K, Shimizu J et al (2020) Real-world effectiveness and safety of nivolumab in patients with non-small cell lung cancer: a multicenter retrospective observational study in Japan. Lung Cancer 140:8-18

22. Nakamura Y, Kitano S, Takahashi A et al (2016) Nivolumab for advanced melanoma: pretreatment prognostic factors and early outcome markers during therapy. Oncotarget 7(47):77404-77415

23. Nishikawa D, Suzuki H, Koide Y et al (2018) Prognostic markers in head and neck cancer patients treated with nivolumab. Cancers (Basel) 10(12):466

24. Cowey CL, Liu FX, Black-Shinn J et al (2018) Pembrolizumab utilization and outcomes for advanced melanoma in US community oncology practices. J Immunother 41(2):86-95

25. Liu FX, Ou W, Diede SJ et al (2019) Real-world experience with pembrolizumab in patients with advanced melanoma: a large retrospective observational study. Medicine (Baltimore) 98(30):e16542

26. Sternberg CN, Loriot Y, James N et al (2019) Primary results from SAUL, a multinational single-arm safety study of atezolizumab therapy for locally advanced or metastatic urothelial or nonurothelial carcinoma of the urinary tract. Eur Urol 76(1):73-81
27. Aspeslagh S, Matias M, Palomar V et al (2017) In the immunooncology era, is anti-PD-1 or anti-PD-L1 immunotherapy modifying the sensitivity to conventional cancer therapies? Eur J Cancer 87:65-74

28. Saleh K, Daste A, Martin N et al (2019) Response to salvage chemotherapy after progression on immune checkpoint inhibitors in patients with recurrent and/or metastatic squamous cell carcinoma of the head and neck. Eur J Cancer 121:123-129

29. Schvartsman G, Peng SA, Bis G et al (2017) Response rates to single-agent chemotherapy after exposure to immune checkpoint inhibitors in advanced non-small-cell lung cancer. Lung Cancer 112:90-95

30. Wakasaki T, Yasumatsu R, Uchi R et al (2020) Outcome of chemotherapy following nivolumab treatment for recurrent and/or metastatic head and neck squamous cell carcinoma. Auris Nasus Larynx 47(1):116-122

31. Weber JS, Hodi FS, Wolchok JD et al (2017) Safety profile of nivolumab monotherapy: a pooled analysis of patients with advanced melanoma. J Clin Oncol 35(7):785-792

Publisher's Note Springer Nature remains neutral with regard to jurisdictional claims in published maps and institutional affiliations.

\section{Affiliations}

\section{Nobuhiro Hanai ${ }^{1} \cdot$ Yasushi Shimizu$^{2} \cdot$ Shin Kariya ${ }^{3} \cdot$ Ryuji Yasumatsu $^{4} \cdot$ Tomoya Yokota $^{5} \cdot$ Takashi Fujii $^{6}$. Kiyoaki Tsukahara ${ }^{7} \cdot$ Masafumi Yoshida $^{8} \cdot$ Kenji Hanyu $^{9}$. Tsutomu Ueda ${ }^{10}$. Hitoshi Hirakawa ${ }^{11}$. Shunji Takahashi ${ }^{12}$. Takeharu Ono ${ }^{13}$. Daisuke Sano ${ }^{14}$. Moriyasu Yamauchi ${ }^{15} \cdot$ Akihito Watanabe $^{16} \cdot$ Koichi Omori $^{17}$. Tomoko Yamazaki ${ }^{18} \cdot$ Nobuya Monden $^{19} \cdot$ Naomi Kudo $^{20} \cdot$ Makoto Arai $^{21} \cdot$ Daiju Sakurai $^{22} \cdot$ Takahiro Asakage $^{23}$. Issei $\mathrm{Doi}^{24} \cdot$ Takayuki Yamada $^{25} \cdot$ Akihiro Homma ${ }^{26}$}

1 Department of Head and Neck Surgery, Aichi Cancer Center Hospital, Nagoya, Japan

2 Department of Medical Oncology, Faculty of Medicine and Graduate School of Medicine, Hokkaido University, Sapporo, Japan

3 Department of Otolaryngology, Head and Neck Surgery, Okayama University Hospital, Okayama, Japan

4 Department of Otolaryngology, Graduate School of Medical Sciences, Kyushu University, Fukuoka, Japan

5 Division of Gastrointestinal Oncology, Shizuoka Cancer Center, Nagaizumi, Japan

6 Department of Head and Neck Surgery, Osaka International Cancer Institute, Osaka, Japan

7 Department of Otorhinolaryngology, Head and Neck Surgery, Tokyo Medical University, Tokyo, Japan

8 Otorhinolaryngology and Head and Neck Surgery, The University of Tokyo Hospital, Tokyo, Japan

9 Head and Neck Oncology Center, International University of Health and Welfare, Mita Hospital, Tokyo, Japan

10 Department of Otorhinolaryngology, Head and Neck Surgery, Hiroshima University Hospital, Hiroshima, Japan

11 Department of Otorhinolaryngology, Head and Neck Surgery, University of the Ryukyu Hospital, Nishihara, Japan
12 Department of Medical Oncology, The Cancer Institute Hospital of Japanese Foundation for Cancer Research, Tokyo, Japan

13 Department of Otolaryngology, Head and Neck Surgery, Kurume University Hospital, Kurume, Japan

14 Department of Otolaryngology, Head and Neck Surgery, Yokohama City University Hospital, Yokohama, Japan

15 Department of Otolaryngology, Head and Neck Surgery, Saga University Hospital, Saga, Japan

16 Department of Otolaryngology, Head and Neck Surgery, Keiyukai Sapporo Hospital, Sapporo, Japan

17 Department of Otolaryngology, Head and Neck Surgery, Kyoto University Hospital, Kyoto, Japan

18 Division of Head and Neck Cancer Oncology, Miyagi Cancer Center, Sendai, Japan

19 Department of Head and Neck Surgery, National Hospital Organization Shikoku Cancer Center, Matsuyama, Japan

20 Department of Otorhinolaryngology, Hirosaki University Graduate School of Medicine, Hirosaki, Japan

21 Department of Medical Oncology, Chiba University Hospital, Chiba, Japan

22 Department of Otorhinolaryngology, Head and Neck Surgery, Chiba University Hospital, Chiba, Japan 
23 Department of Head and Neck Surgery, Tokyo Medical and Dental University Medical Hospital, Tokyo, Japan

24 Medical Affairs, ONO Pharmaceutical Co., Ltd, Osaka, Japan

25 Japan Medical and Development, Bristol-Myers Squibb K.K., Tokyo, Japan
26 Department of Otolaryngology, Head and Neck Surgery, Faculty of Medicine and Graduate School of Medicine, Hokkaido University, Kita15 Nishi7, Kita-Ku, Sapporo, Hokkaido 060-8638, Japan 